\title{
A note on common fixed point theorems in partial metric spaces
}

\author{
Erdal Karapinar
}




\title{
A NOTE ON COMMON FIXED POINT THEOREMS IN PARTIAL METRIC SPACES
}

\author{
ERDAL KARAPINAR
}

Received February 16, 2011

\begin{abstract}
In this manuscript, we consider the notion of generalized Sehgal contraction condition in a partial metric space. For the pair of two self mappings $(S, T)$ which satisfies Sehgal contraction condition, we obtain a unique common fixed point.
\end{abstract}

2000 Mathematics Subject Classification: 46N40; 47H10; 54H25; 46 T99

Keywords: partial metric space, Sehgal contraction condition, common fixed point

\section{INTRODUCTION AND PRELIMINARIES}

Partial metric space (in short PMS), is one of the attempts to generalize the notion of the metric space that by replacing the condition $d(x, x)=0$ with the condition $d(x, x) \leq d(x, y)$ for all $x, y$ in the definition of the metric (see e.g. $[14,15])$. In these initial papers, Matthews discussed not only the general topological properties of partial metric spaces but also some properties of convergence of sequences. In $[14,15]$, he proved a fixed point theorem for contractive mappings of partial metric spaces: Any mapping $T$ of a complete partial metric space $X$ into itself that satisfies, for some $0 \leq k<1$, the inequality $d(T x, T y) \leq k d(x, y)$, for all $x, y \in X$, has a unique fixed point. Recently, many authors (see e.g.[1,2, 8, 10-17, 20]) focused on this subject and generalized some fixed point theorems from the class of metric spaces to the class of partial metric spaces. In this manuscript, we discuss existence and uniqueness of a common fixed point of self-mappings $S, T$ of partial metric spaces.

A partial metric space (See e.g. $[14,15])$ is a pair $\left(X, p: X \times X \rightarrow \mathbb{R}^{+}\right)\left(\right.$where $\mathbb{R}^{+}$ denotes the set of all non negative real numbers) such that

(PM1) $p(x, y)=p(y, x)$ (symmetry)

(PM2) If $0 \leq p(x, x)=p(x, y)=p(y, y)$ then $x=y$ (equality)

(PM3) $p(x, x) \leq p(x, y)$ (small self-distances)

(PM4) $p(x, z)+p(y, y) \leq p(x, y)+p(y, z)$ (triangularity)

for all $x, y, z \in X$. For a partial metric $p$ on $X$, the functions $d_{p}, d_{m}: X \times X \rightarrow \mathbb{R}^{+}$ given by

$$
d_{p}(x, y)=2 p(x, y)-p(x, x)-p(y, y)
$$


and

$$
d_{m}(x, y)=\max \{p(x, y)-p(x, x), p(x, y)-p(y, y)\}
$$

are (usual) metrics on $X$. It is clear that $d_{p}$ and $d_{m}$ are equivalent. Each partial metric $p$ on $X$ generates a $T_{0}$ topology $\tau_{p}$ on $X$ with a base consisting of the family of open $p$-balls $\left\{B_{p}(x, \varepsilon): x \in X, \varepsilon>0\right\}$, where $B_{p}(x, \varepsilon)=\{y \in X: p(x, y)<p(x, x)+\varepsilon\}$ for all $x \in X$ and $\varepsilon>0$.

Example 1. A basic example of partial metric is $\left(\mathbb{R}^{+}, p\right)$, where $p(x, y)=\max \{x, y\}$. It is clear that $p$ is not a (usual) metric. Note that in this case $d_{p}(x, y)=|x-y|$ and $d_{m}(x, y)=\frac{1}{2}|x-y|$.

Example 2. (See [8]) Let $X=\{[a, b]: a, b, \in \mathbb{R}, a \leq b\}$ and define $p([a, b],[c, d])$ $=\max \{b, d\}-\min \{a, c\}$. Then $(X, p)$ is a partial metric spaces.

Example 3. (See [8]) Let $X:=[0,1] \cup[2,3]$ and define $p: X \times X \rightarrow[0, \infty)$ by $p(x, y)=\left\{\begin{array}{c}\max \{x, y\} \text { if }\{x, y\} \cap[2,3] \neq \varnothing \\ |x-y| \text { if }\{x, y\} \subset[0,1] .\end{array}\right.$

Then $(X, p)$ is a complete partial metric space.

Definition 1. (See e.g. [14,15])

(i) A sequence $\left\{x_{n}\right\}$ in a PMS $(X, p)$ converges to $x \in X$ if and only if $p(x, x)=$ $\lim _{n \rightarrow \infty} p\left(x, x_{n}\right)$,

(ii ) a sequence $\left\{x_{n}\right\}$ in a PMS $(X, p)$ is called a Cauchy if and only if $\lim _{n, m \rightarrow \infty} p\left(x_{n}, x_{m}\right)$ exists (and finite),

(iii) A PMS $(X, p)$ is said to be complete if every Cauchy sequence $\left\{x_{n}\right\}$ in $X$ converges, with respect to $\tau_{p}$, to a point $x \in X$ such that $p(x, x)=\lim _{n, m \rightarrow \infty} p\left(x_{n}, x_{m}\right)$.

(iv) A mapping $f: X \rightarrow X$ is said to be continuous at $x_{0} \in X$, if for every $\varepsilon>0$, there exists $\delta>0$ such that $f\left(B\left(x_{0}, \delta\right)\right) \subset B\left(f\left(x_{0}\right), \varepsilon\right)$.

Lemma 1. (See e.g. [14, 15])

(A) A sequence $\left\{x_{n}\right\}$ is Cauchy in a PMS $(X, p)$ if and only if $\left\{x_{n}\right\}$ is Cauchy in a metric space $\left(X, d_{p}\right)$,

(B) A PMS $(X, p)$ is complete if and only if a metric space $\left(X, d_{p}\right)$ is complete. Moreover,

$$
\lim _{n \rightarrow \infty} d_{p}\left(x, x_{n}\right)=0 \Leftrightarrow p(x, x)=\lim _{n \rightarrow \infty} p\left(x, x_{n}\right)=\lim _{n, m \rightarrow \infty} p\left(x_{n}, x_{m}\right)
$$

Remark 1. Since $d_{p}$ and $d_{m}$ are equivalent, we can take $d_{p}$ instead of $d_{m}$ in this Lemma.

Let $(X, p)$ be a PMS and denote the closure of the set $\{p(x, y): x, y \in X\}$ by $P$ and $P^{3}=P \times P \times P$. A function $\phi: P^{3} \rightarrow \mathbb{R}^{+}$is right continuous if and only if

(S1) the sequences $\left\{a_{n}\right\},\left\{b_{n}\right\},\left\{c_{n}\right\}$ decrease and converge to $a, b, c \in P$, respectively, 
then

$$
\phi\left(a_{n}, b_{n}, c_{n}\right) \rightarrow \phi(a, b, c) .
$$

The function $\phi$ is called symmetric if and only if

$$
\phi(a, b, c)=\phi(b, a, c) \text {, for all }(a, b, c) \in P^{3} .
$$

In the spirit of Sehgal [19], we state the following definition for partial metric spaces.

Definition 2. Let $(X, p)$ be a PMS and $S, T: X \rightarrow X$ be two mappings. The pair $(S, T)$ is said to satisfy Sehgal k-condition if and only if there are maps $I_{S}: S \times X \rightarrow$ $\mathbb{Z}^{+}$and $I_{T}: T \times X \rightarrow \mathbb{Z}^{+}$such that if $r(x)=I_{S}(S, x)$ and $q(x)=I_{T}(T, x)$, then

$$
p\left(S^{r(x)} x, T^{q(y)} y\right) \leq k \phi\left(p\left(S^{r(x)} x, x\right), p\left(y, T^{q(y)} y\right), p(x, y)\right)
$$

for all $x, y \in X$, where $k \in \mathbb{R}$ and $\phi$ is a symmetric right continuous. If $0 \leq k<1$, then we say that $(S, T)$ satisfy Sehgal contraction condition.

\section{MAIN RESULTS}

The following two lemmas are easy to prove but they will be very useful in the proof of the main theorem.

Lemma 2. (See e.g. $[1,10])$ Let $(X, p)$ be a complete PMS. Then

(A) If $p(x, y)=0$ then $x=y$,

(B) If $x \neq y$, then $p(x, y)>0$.

Lemma 3. (See e.g. $[1,10])$ Assume $x_{n} \rightarrow z$ as $n \rightarrow \infty$ in a PMS $(X, p)$ such that $p(z, z)=0$. Then $\lim _{n \rightarrow \infty} p\left(x_{n}, y\right)=p(z, y)$ for every $y \in X$.

The following theorem extends the results of [19].

Theorem 1. Let $(X, p)$ be a complete partial metric space. Suppose that $S, T$ : $X \rightarrow X$ are two mappings such that the pair $(S, T)$ satisfies Sehgal's contraction contraction.

(A) If $\phi(a, b, c) \leq \max \{a, b, c\}$, for $(a, b, c) \in P^{3}$, then $S$ and $T$ have a unique common fixed point in $X$, that is, $S^{r(z)} z=T^{q(z)} z=z$.

Proof. Let $x_{0} \in X$. Define the sequence $\left\{x_{n}\right\}_{n=1}^{\infty}$ in a way that $x_{2}=T^{q\left(x_{1}\right)} x_{1}$ and $x_{1}=S^{r\left(x_{0}\right)} x_{0}$ and inductively

$$
x_{2 n+2}=T^{q\left(x_{2 n+1}\right)} x_{2 n+1} \text { and } x_{2 n+1}=S^{r\left(x_{2 n}\right)} x_{2 n} \text { for } n=0,1,2, \ldots
$$

If $n$ is odd, due to (1.4), we have

$$
p\left(x_{n+1}, x_{n+2}\right)=p\left(T x_{n}, S x_{n+1}\right) \leq k \phi\left(p\left(x_{n}, x_{n+1}\right), p\left(x_{n+1}, x_{n+2}\right), p\left(x_{n}, x_{n+1}\right)\right)
$$


Regarding the assumption of $(A)$,

$$
p\left(x_{n+1}, x_{n+2}\right)=p\left(T x_{n}, S x_{n+1}\right) \leq k \max \left\{p\left(x_{n}, x_{n+1}\right), p\left(x_{n+1}, x_{n+2}\right)\right\}
$$

If $\max \left\{p\left(x_{n}, x_{n+1}\right), p\left(x_{n+1}, x_{n+2}\right)\right\}=p\left(x_{n+1}, x_{n+2}\right)$ then the expression (2.2) turns into

$$
p\left(x_{n+1}, x_{n+2}\right) \leq k p\left(x_{n+1}, x_{n+2}\right) .
$$

Since $k<1$, this is impossible. Thus, we have

$$
p\left(x_{n+1}, x_{n+2}\right) \leq k p\left(x_{n}, x_{n+1}\right) .
$$

If $n$ is even, analogously we observe that $p\left(x_{n+1}, x_{n+2}\right) \leq k p\left(x_{n}, x_{n+1}\right)$. Observe that $\left\{p\left(x_{n}, x_{n+1}\right)\right\}$ is a non-negative, non-increasing sequence of reals. Regarding (2.3), one can observe that

$$
p\left(x_{n}, x_{n+1}\right) \leq k^{n} p\left(x_{0}, x_{1}\right), \forall n=0,1,2, \cdots
$$

Letting $n \rightarrow \infty$, the right hand side of (2.4) tends to zero.

Consider now

$$
\begin{aligned}
d_{p}\left(x_{n+1}, x_{n+2}\right) & =2 p\left(x_{n+1}, x_{n+2}\right)-p\left(x_{n+1}, x_{n+1}\right)-p\left(x_{n+2}, x_{n+2}\right) \\
& \leq 2 p\left(x_{n+1}, x_{n+2}\right) \\
& \leq 2 k^{n+1} p\left(x_{0}, x_{1}\right) .
\end{aligned}
$$

Hence, regarding (2.4), we have $\lim _{n \rightarrow \infty} d_{p}\left(x_{n+1}, x_{n+2}\right)=0$. Moreover,

$$
\begin{aligned}
d_{p}\left(x_{n+1}, x_{n+s}\right) & \leq d_{p}\left(x_{n+s-1}, x_{n+s}\right)+\cdots+d_{p}\left(x_{n+1}, x_{n+2}\right) \\
& \leq 2 k^{n+s} p\left(x_{0}, x_{1}\right)+\cdots+2 k^{n+1} p\left(x_{0}, x_{1}\right)
\end{aligned}
$$

which implies that $\left\{x_{n}\right\}$ is a Cauchy sequence in $\left(X, d_{p}\right)$ that is, $d_{p}\left(x_{n}, x_{m}\right) \rightarrow 0$. Since $(X, p)$ is complete, by Lemma $1.3,\left(X, d_{p}\right)$ is complete and the sequence $\left\{x_{n}\right\}$ is convergent in $\left(X, d_{p}\right)$, say to $z \in X$.

By Lemma 1.3,

$$
p(z, z)=\lim _{n \rightarrow \infty} p\left(x_{n}, z\right)=\lim _{n, m \rightarrow \infty} p\left(x_{n}, x_{m}\right)
$$

Since $\left\{x_{n}\right\}$ is a Cauchy sequence in $\left(X, d_{p}\right)$, we have $\lim _{n, m \rightarrow \infty} d_{p}\left(x_{n}, x_{m}\right)=0$. Since

$$
\max \left\{p\left(x_{n}, x_{n}\right), p\left(x_{n+1}, x_{n+1}\right)\right\} \leq p\left(x_{n}, x_{n+1}\right),
$$

then by (2.4), it follows that

$$
\max \left\{p\left(x_{n}, x_{n}\right), p\left(x_{n+1}, x_{n+1}\right)\right\} \leq k^{n+1} p\left(x_{0}, x_{1}\right)
$$

Thus from (2.4), (2.8) and from the definition of $d_{p}$, we have $\lim _{n, m \rightarrow \infty} p\left(x_{n}, x_{m}\right)=0$. Therefore from (2.7) we have

$$
p(z, z)=\lim _{n \rightarrow \infty} p\left(x_{n}, z\right)=\lim _{n, m \rightarrow \infty} p\left(x_{n}, x_{m}\right)=0 .
$$


We assert that $T^{q(z)} z=z$. Assume $T^{q(z)} z \neq z$, then $p\left(z, T^{q(z)} z\right)>0$. Let $\left\{x_{2 n(i)}\right\}$ be subsequence of $\left\{x_{2 n}\right\}$ and hence $\left\{x_{n}\right\}$. Due to (PM4), we have

$$
\begin{gathered}
p\left(S x_{2 n(i)}, T^{q(z)} z\right)=p\left(S x_{2 n(i)}, T^{q(z)} z\right) \\
\leq k \phi\left(p\left(x_{2 n(i)}, x_{2 n(i)+1}\right), p\left(T^{q(z)} z, z\right), p\left(x_{2 n(i)}, z\right)\right.
\end{gathered}
$$

Letting $n \rightarrow \infty$ and taking the assumption of $(A)$ and (2.9) into account, we get that

$$
p\left(z, T^{q(z)} z\right) \leq k \phi\left(0, p\left(T^{q(z)} z, z\right), 0\right) \leq k p\left(T^{q(z)} z, z\right)
$$

Since $k<1$, then $p\left(T^{q(z)} z, z\right)=0$. By Lemma 2, we get $T^{q(z)} z=z$. Analogously, if we choose a subsequence $\left\{x_{2 n(i)+1}\right\}$ be subsequence of $\left\{x_{2 n+1}\right\}$, we obtain $S^{r(z)} z=z$.

Assume now that there exists $w \in X$ such that $S^{r(w)}=T^{q(w)} w=w$. By (PM3)

$$
p(z, z) \leq p(z, w) \text { and } p(w, w) \leq p(z, w)
$$

Regarding that the function $\phi$ satisfies the condition of $(A)$ with (2.12), we get

$$
\begin{aligned}
p(z, w)=p\left(S^{r(z)} z, T^{q(w)} w\right) & \leq k \phi\left(p\left(z, S^{r(z)} z\right), p\left(T^{q(w)} w, w\right), p(z, w)\right) \\
& \leq k \phi(p(z, z), p(w, w), p(z, w)) \\
& \leq k p(z, w)
\end{aligned}
$$

Since $k<1$, it yields a contradiction.

Thus, $p(z, w)=0$ and by Lemma 2 we have $z=w$.

Corollary 1. Let $(X, p)$ be a complete partial metric space. Suppose $I_{T}$ and $I_{S}$ are defined as above. $S, T: X \rightarrow X$ are two mappings such that the pair $(S, T)$ satisfies one of the following condition:

(A) $p\left(S^{r(x)} x, T^{q(y)} y\right) \leq k \max \left\{p\left(S^{r(x)} x, x\right), p\left(y, T^{q(y)} y\right), p(x, y)\right\}$ for some $0 \leq k<1$,

(B) $p\left(S^{r(x)} x, T^{q(y)} y\right) \leq \alpha p\left(S^{r(x)} x, x\right)+\beta p\left(y, T^{q(y)} y\right)+\gamma p(x, y)$ for some non-negative reals $\alpha, \beta, \gamma$ with $\alpha+\beta+\gamma<1$.

Then $S$ and $T$ have a unique common fixed point in $X$, that is, $S^{r(z)} z=T^{q(z)} z=$ $z$.

Proof. For $(A)$, we choose a function $\phi(a, b, c)=\max \{a, b, c\}$ as in Theorem 1 . In case of $(B)$, set $k=\alpha+\beta+\gamma$. Then $(A)$ implies $(B)$.

Notice that this corollary generalizes also some results of ([6] -[4]).

Corollary 2. Let $(X, p)$ be a complete partial metric space. Let $S, T: X \rightarrow X$ be two mappings such that the pair $(S, T)$ satisfies the following condition:

$$
p\left(S^{r} x, T^{q} y\right) \leq k \phi\left(p\left(S^{r} x, x\right), p\left(y, T^{q} y\right), p(x, y)\right)
$$

for all $x, y \in X$ where $0 \leq k<1$ and $\phi$ is symmetric right-continuous. If $\phi(a, b, c) \leq$ $\max \{a, b, c\}$ then $S$ and $T$ have a unique common fixed point theorem. 
Proof. By Theorem 1, and by taking the maps $I_{T}, I_{S}$ as a constant, we get that $S^{r}$ and $T^{q}$ have a unique common fixed point, say $z \in X$. Now consider

$$
S^{q}(S z)=S^{q+1} z=S\left(S^{q} z\right)=S z
$$

which says that $S z$ is a fixed point of $S^{q}$. Since $z$ is the unique fixed point of $S^{q}$, then $S z=z$. Analogously, one can get $T z=z$.

Corollary 3. Let $(X, p)$ be a complete partial metric space. Let $S, T: X \rightarrow X$ be two mappings such that the pair $(S, T)$ satisfies the following condition:

$$
p\left(S^{r} x, T^{q} y\right) \leq k \max \left\{p\left(S^{r} x, x\right), p\left(y, T^{q} y\right), p(x, y)\right\}
$$

for all $x, y \in X$ where $0 \leq k<1$ and $\phi$ is symmetric right-continuous. If $\phi(a, b, c) \leq$ $\max \{a, b, c\}$ then $S$ and $T$ have a unique common fixed point theorem.

Corollary 4. Let $(X, p)$ be a complete partial metric space. Let $S, T: X \rightarrow X$ be two mappings such that the pair $(S, T)$ satisfies the following condition:

$$
p\left(S^{r} x, T^{q} y\right) \leq \alpha p\left(S^{r} x, x\right)+\beta p\left(y, T^{q} y\right)+\gamma p(x, y)
$$

for all $x, y \in X$, where for some non-negative reals $\alpha, \beta, \gamma$ with $\alpha+\beta+\gamma<1.0 \leq$ $k<1$ and $\phi$ is symmetric right-continuous. If $\phi(a, b, c) \leq \max \{a, b, c\}$ then $S$ and $T$ have a unique common fixed point theorem.

Remark 2. Consider Corollary 4 and take $S=T$.

(1) If we set $r=q$ in (2.15) then we get Reich type fixed point theorem (See e.g. $[2,18])$.

(2) If we set $r=q=1$ and $\gamma=0$ in (2.15) we get Kannan type fixed point theorem (See e.g. [2,9] )

(3) If we set $r=q=1$ and $\alpha=\beta=0$ in (2.15) we get Banach type fixed point theorem (See e.g. [2,3, 15, 17] and [4-7])

Example 4. Let $X=[0,1]$ and $p(x, y)=\max \{x, y\}$. It is clear that $(X, p)$ is a partial metric spaces but not a metric. Suppose that $S x=T x=\frac{x}{2}$ and $I_{S}, I_{T}$ are constant mappings, such as $r(x)=2=q(y)$. Take $\phi(a, b, c)=\frac{1}{3}[a+b+c]$. Let $p(x, y)=\max \{x, y\}$ for all $x, y \in X$. For $\frac{k}{3}$ the condition of Corollary 2 is satisfied. Clearly, 0 is the common fixed point of $S, T$.

Example 5. Let $X=[1,15]$ and $p(x, y)=\max \{x, y\}$. Here $(X, p)$ is a complete metric spaces. Define the self-mappings $S, T: X \rightarrow X$ as $T x=\frac{x^{2}}{1+x}$ and $S x=$ $\left\{\begin{array}{c}\frac{x}{1+x} \text { if } 1<x \leq 15 \\ 0 \text { if } 0 \leq x \leq 1\end{array}\right.$. Set $\phi(a, b, c)=\frac{19}{20} \max \{x, y\}$. Without loss of generality, assume $y<x$. Thus, $p(T x, x)=x, p(x, y)=x, p(S y, y)=y$ and $p(T x, S y)=$ $\frac{x^{2}}{1+x}$. Clearly, $p(T x, S y)=\frac{x^{2}}{1+x} \leq \phi(x, y, x)=\frac{19}{20} x$. Hence, it satisfies the conditions of Corollary 2.14 for $r=1$ and $q=1$, and 0 is the unique common fixed point of $S$ and $T$. 


\section{REFERENCES}

[1] T. Abdeljawad, E. Karapınar, and K. Taş, "Existence and uniqueness of a common fixed point on partial metric spaces," Appl. Math. Lett., vol. 24, no. 11, pp. 1900-1904, 2011.

[2] I. Altun, F. Sola, and H. Simsek, "Generalized contractions on partial metric spaces," Topology Appl., vol. 157, no. 18, pp. 2778-2785, 2010.

[3] S. Banach, "Surles operations dans les ensembles abstraits et leur application aux equations itegrales," Fund. Math., vol. 3, pp. 133-181, 1922.

[4] V. Berinde, "Some remarks on a fixed point theorem for Ćirić-type almost contractions," Carpathian J. Math., vol. 25, no. 2, pp. 157-162, 2009.

[5] V. Berinde, "Approximating common fixed points of noncommuting almost contractions in metric spaces," Fixed Point Theory, vol. 11, no. 2, pp. 179-188, 2010.

[6] V. Berinde, "Common fixed points of noncommuting almost contractions in cone metric spaces," Math. Commun., vol. 15, no. 1, pp. 229-241, 2010.

[7] V. Berinde, "Common fixed points of noncommuting discontinuous weakly contractive mappings in cone metric spaces," Taiwanese J. Math., vol. 14, no. 5, pp. 1763-1776, 2010.

[8] D. Ilić, V. Pavlović, and V. Rakočević, "Some new extensions of Banach's contraction principle to partial metric space," Appl. Math. Lett., vol. 24, no. 8, pp. 1326-1330, 2011.

[9] R. Kannan, "Some results on fixed points," Bull. Calcutta Math. Soc., vol. 60, pp. 71-76, 1968.

[10] E. Karapınar, "Generalizations of Caristi Kirk's theorem on partial metric spaces," Fixed Point Theory Appl., vol. 2011, no. 2011:4, p. 7, 2011.

[11] E. Karapınar, "Weak $\varphi$-contraction on partial metric spaces and existence of fixed points in partially ordered sets," Math. Aterna, vol. 1, no. 3-4, pp. 237-244, 2011.

[12] E. Karapınar, "Weak $\varphi$-contraction on partial metric spaces," J. Comput. Anal. Appl., (in press).

[13] E. Karapınar and I. M. Erhan, "Fixed point theorems for operators on partial metric spaces," Appl. Math. Lett., vol. 24, no. 11, pp. 1894-1899, 2011.

[14] S. G. Matthews, "Partial metric topology," in Research Report 212. Dept. of Computer Science. University of Warwick, 1992.

[15] S. G. Matthews, "Partial metric topology," in Papers on general topology and applications, ser. Ann. New York Acad. Sci., vol. 728. New York: New York Acad. Sci., 1994, pp. 183-197.

[16] S. Oltra, S. Romaguera, and E. A. Sánchez-Pérez, "The canonical partial metric and the uniform convexity on normed spaces," Appl. Gen. Topol., vol. 6, no. 2, pp. 185-194, 2005.

[17] S. Oltra and O. Valero, "Banach's fixed point theorem for partial metric spaces," Rend. Istit. Mat. Univ. Trieste, vol. 36, no. 1-2, pp. 17-26, 2004.

[18] S. Reich, "Kannan's fixed point theorem," Boll. Un. Mat. Ital., vol. 4, no. 4, pp. 1-11, 1971.

[19] V. M. Sehgal, "Some fixed and common fixed point theorems in metric spaces," Canad. Math. Bull., vol. 17, pp. 257-259, 1974.

[20] O. Valero, "On Banach fixed point theorems for partial metric spaces," Appl. Gen. Topol., vol. 6, no. 2, pp. 229-240, 2005.

\section{Author's address}

\section{Erdal Karapınar}

Atılım University, Department of Mathematics, İncek, 06836 Ankara, Turkey

E-mail address: erdalkarapinar@yahoo.com; ekarapinar@atilim.edu.tr 